\title{
Modes and nodes explain the mechanism of action of vortioxetine, a multimodal agent (MMA): enhancing serotonin release by combining serotonin (5HT) transporter inhibition with actions at $5 \mathrm{HT}$ receptors (5HT1A, 5HT1B, 5HT1D, 5HT7 receptors)
}

\author{
Stephen M. Stahl
}

ISSUE:

Vortioxetine is an antidepressant that targets multiple pharmacologic modes of action at sites - or nodes - where serotonergic neurons connect to various brain circuits. These multimodal pharmacologic actions of vortioxetine lead to enhanced release of various neurotransmitters, including serotonin, at various nodes within neuronal networks.

\section{Take-Home Points}

- Most known antidepressants, including vortioxetine, inhibit the serotonin (5HT) transporter (SERT) as one of their pharmacologic actions.

- Although SERT inhibition enhances 5HT release, that release is blunted at various nodes within neuronal networks when $5 \mathrm{HT}$ stimulates $5 \mathrm{HT}$ negative feedback receptors.

- More robust release of $5 \mathrm{HT}$ can be caused by vortioxetine, even at lower degrees of SERT inhibition, when 5HT1B, 5HT1D, and 5HT7 receptors are also fully or partially blocked, and if $5 \mathrm{HT} 1 \mathrm{~A}$ receptors are also stimulated at nodes within brain circuits where serotonin neurons connect to neural networks

Five modes of action currently describe the range of pharmacologic targets for psychotropic drugs ${ }^{1-3}$ :

1. Inhibition of transporters

2. Actions at $\mathrm{G}$ protein-linked receptors, from stimulation to blockade
3. Actions at ligand-gated ion channels, from stimulation to blockade

4. Actions at voltage-gated ion channels

5. Enzyme inhibition

Most psychotropic drugs have a single mode of action, even if they have multiple targets within that mode. ${ }^{1-3}$ That is, some antidepressants block more than one transporter. ${ }^{2,3}$ Also, essentially all antipsychotics block more than one $G$ protein-linked receptor. ${ }^{2,3}$ However, only a few psychotropic drugs target more than one mode of action. Vortioxetine is an example of such a "multimodal" agent, since it targets 3 modes of action (Figure 1) ${ }^{1-6}$ :

1. Inhibition of a transporter (ie, the serotonin transporter or SERT)

2. Actions at several G-protein linked receptors (ie, agonist actions at 5HT1A receptors, partial agonist actions at 5HT1B receptors, antagonist actions at 5HT1D and 5HT7 receptors)

3. Inhibition of a ligand-gated ion channel (the 5HT3 receptor) 
Figure 1. Icon of vortioxetine showing its 6 pharmacologic mechanisms. Highlighted here are the 5 actions principally linked to regulation of serotonin $(5 \mathrm{HT})$ release, namely serotonin transporter (SERT) inhibition; 5HT1A agonism; 5HT1B partial agonism; and 5HT1D and 5HT7 antagonism.

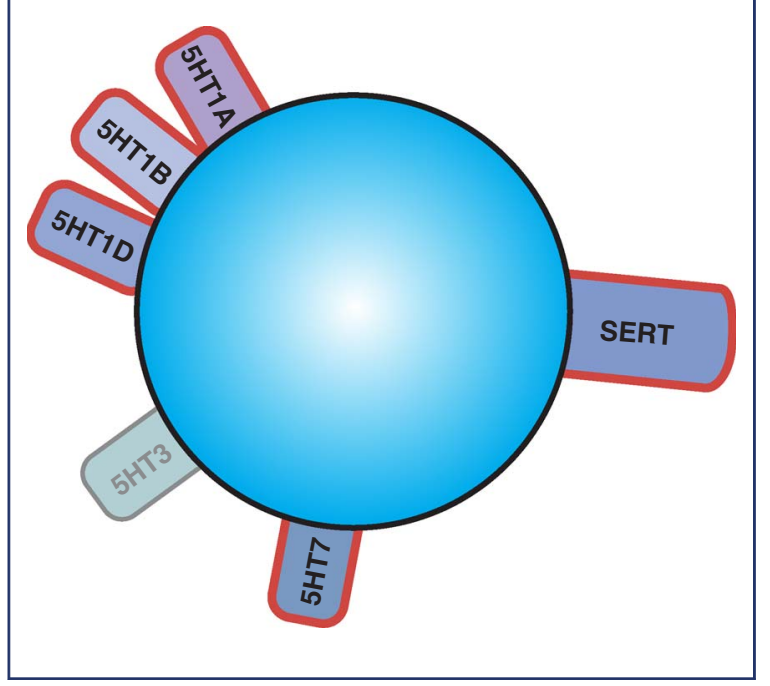

Although vortioxetine thus targets a complex mixture of pharmacologic modes, the combined effects can be conceptualized more simply as actions that work together to alter the downstream release of numerous neurotransmitters. Here, the mechanism whereby these receptors work together to enhance the release of serotonin is described. The mechanism whereby these $5 \mathrm{HT}$ receptors work together to alter the release of other neurotransmitters is covered in other Brainstorms articles. $^{7-9}$

\section{Modes and Nodes}

Actions of vortioxetine can be understood not only within "microcircuits" or synapses between presynaptic 5HT neurons and post synaptic sites, but also within "macrocircuits" where serotonergic neurons are part of a neuronal network that connects many neurons with each other. Sometimes the sites where neurons connect at synapses are also called the "nodes" of a neuronal network. Nodes are distributed anatomically throughout the brain and can sometimes be visualized with modern neuroimaging techniques. ${ }^{2}$ Since there is topographical localization of function within the brain, one network of brain circuits can regulate entirely different functions than another. Thus different functions, such as cognition, fear, reward, and arousal, each map to different brain circuits and brain regions. ${ }^{1,2,10}$
The entire wiring diagram of the brain is also called the connectome, and changes in neurotransmitter release within the connectome theoretically alter the "strength" of connectivity of one brain area to the next.

Thus, drugs such as vortioxetine and many others that alter the release of neurotransmitters can modify the connectivity of the brain and thereby potentially reduce symptoms by changing the efficiency of information processing in specific brain circuits. Since there are numerous symptoms in any given psychiatric condition, it is likely that there are numerous networks with altered connectivity involved in every psychiatric disorder. Agents that can change more than one neurotransmitter's release in more than one site (ie, multiple modes of action at multiple nodes within brain networks) at least theoretically have the possibility of changing multiple symptoms linked to multiple circuits. ${ }^{2,11,12}$ Thus, it is useful to understand the net effect of the multiple, simultaneous actions of vortioxetine on neurotransmitter release in order to gain insight into its pharmacologic mechanism of action.

\section{Serotonin Release and SERT Inhibition}

Serotonin neurons project widely throughout the brain and interact with many brain circuits via presynaptic nerve terminals containing the well-known SERTs (Figure 2A). 2,13 What may be less widely appreciated are all the negative feedback mechanisms within brain networks that act to limit the amount of 5HT released even when SERTs are inhibited (Figure 2A). These negative feedback mechanisms serve as "brakes" upon the release of $5 \mathrm{HT}$, even in the presence of SERT inhibition. They include 5 HT1A receptors, ${ }^{15-18}$ which are located presynaptically on the soma and dendrites of 5HT neurons, as well as the negative feedback autoreceptor subtypes 5HT1B and 5HT1D, which are located on the presynaptic nerve terminals of $5 \mathrm{HT}$ neurons (Figure 2A). ${ }^{13,17-21}$ Yet another negative feedback upon $5 \mathrm{HT}$ release is $5 \mathrm{HT} 7$ receptors that innervate gamma-amino butyric acid (GABA) neurons in the raphe; when $5 \mathrm{HT}$ stimulates these $5 \mathrm{HT} 7$ receptors, this causes the release of inhibitory GABA, which shuts off further 5HT release (Figure 2A). ${ }^{13,17,22,23}$

When SERTs are inhibited, this causes 5HT to rise everywhere there are presynaptic $5 \mathrm{HT}$ nerve terminals (Figure 2B). However, the amount of 5HT release is mitigated by the multiple negative feedback receptors. To the extent that long-term SERT inhibition desensitizes these receptors, their ability to diminish serotonin release is blunted. This is seen especially with presynaptic somatodendritic 5HT1A receptors, whose 


\section{BRAINSTORMS-Clinical Neuroscience Update}

Figure 2A. Baseline. This figure shows how 5HT release is regulated. 5HT neurons have numerous negative feedback mechanisms within brain networks that act to limit the amount of $5 \mathrm{HT}$ released. These negative feedback mechanisms serve as "brakes" upon the release of 5HT, even in the presence of SERT inhibition. They include 5HT1A receptors located presynaptically on the soma and dendrites of 5HT neurons, as well as the negative feedback autoreceptor subtypes 5HT1B and 5HT1D located on the presynaptic terminals of 5HT neurons. Another negative feedback mechanism for 5HT release is the 5HT7 receptor that innervates gamma amino butyric acid (GABA) neurons in the raphe. When 5HT stimulates these 5HT7 receptors, this causes the release of inhibitory GABA, which shuts off further $5 \mathrm{HT}$ release.

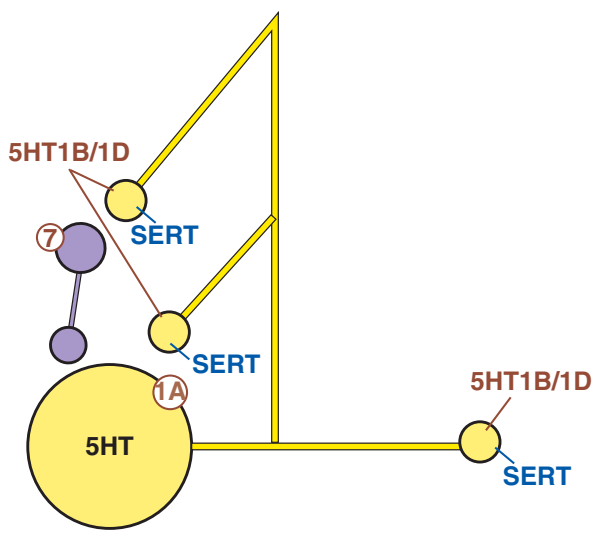

Figure 2B. SERT inhibition. When SERTs (highlighted on the vortioxetine icon) are inhibited (shown as cancelled out with the red symbol at the 5HT nerve terminal), this causes 5HT to rise everywhere there are presynaptic 5HT nerve terminals (indicated by enhanced clouds of yellow 5HT release here compared to Figure 2A). However, the amount of 5HT release is limited due to the multiple negative feedback mechanisms that inhibit further 5HT release. Specifically, presynaptic somatodendritic 5HT1A receptors inhibit 5HT release, especially immediately after administration, but eventually downregulate to allow enhanced release by SERT inhibition with chronic administration. Simultaneous stimulation of 5HT1A presynaptic autoreceptors while blocking SERT may lead to more rapid desensitization of the 5HT1A presynaptic receptors, and make the enhanced release of 5HT occur faster. However, 5HT release is also importantly regulated by 5HT1B, 5HT1D, and 5HT7 receptors, which act long-term to limit the amount of 5HT that is released by SERT inhibition.

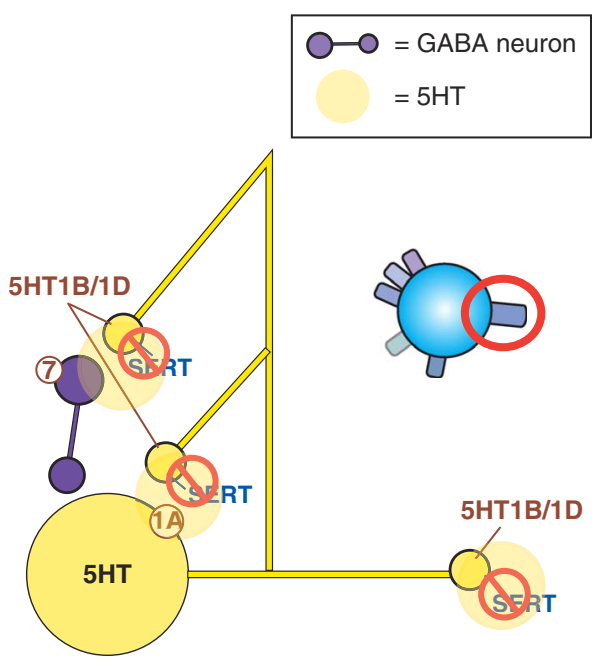


Figure 2C. SERT inhibition combined with stimulating 5HT1A receptors and partially or fully blocking 5HT1B/D and 5HT7 receptors. Shown here are the combination of vortioxetine's actions that lead to enhanced release of 5HT. In addition to

blocking SERT (faded red symbols), vortioxetine simultaneously stimulates 5HT1A receptors and blocks 5HT1B, 5HT1D, and 5HT7 receptors (highlighted on the vortioxetine icon). The negative feedback of 5HT1B, 5HT1D, and 5HT7 receptors is blocked (shown as cancelled out with the bright red symbols at the 5HT nerve terminals), thereby enhancing 5HT release compared to blocking SERT alone (compare yellow cloud of 5HT here with Figure 2B)

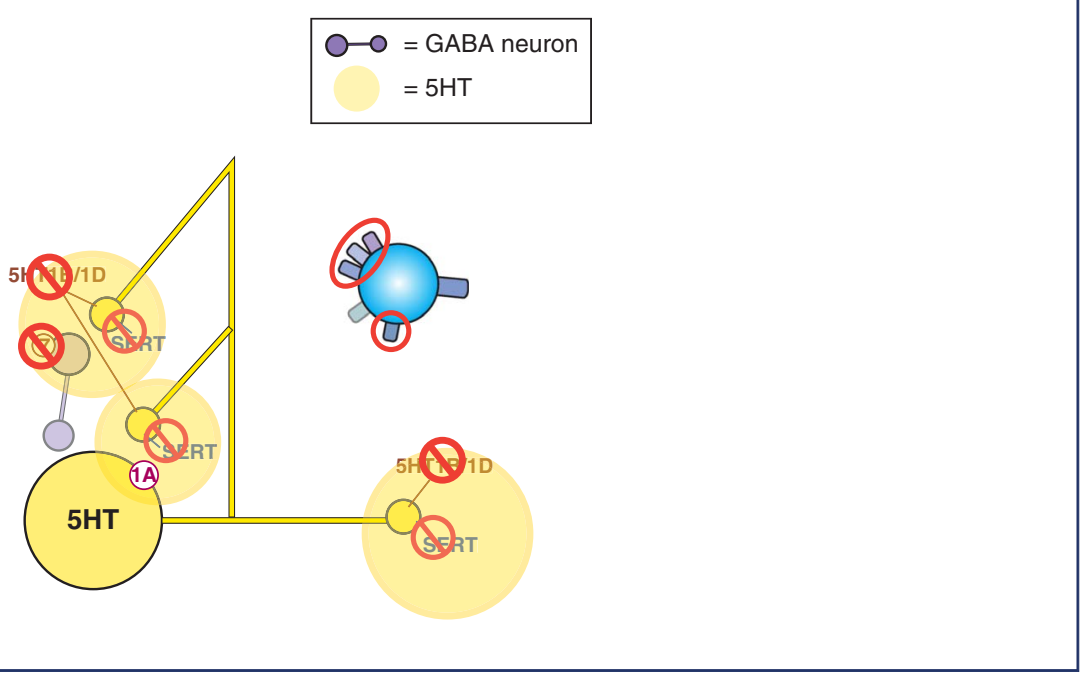

downregulation is considered key to the enhanced release of 5HT caused by SERT inhibition (Figure 2B). What would happen if you simultaneously stimulate those 5HT1A presynaptic autoreceptors on the serotonin cell body and dendrites while blocking SERT? Preclinical evidence suggests that this hastens the desensitization of 5HT1A presynaptic receptors, and makes serotonin release occur faster. ${ }^{14-17}$

Although the role of $5 \mathrm{HT} 1 \mathrm{~A}$ receptors in the regulation of $5 \mathrm{HT}$ release after SERT inhibition is well known, only recently has it become widely recognized that $5 \mathrm{HT}$ release is also importantly regulated by $5 \mathrm{HT}$ actions on 5HT1B, 5HT1D, and 5HT7 receptors (Figure 2B). What happens if you block these receptors while simultaneously blocking SERT (Figure 2C)? Thus, full or partial antagonism of 5HT1B, 5HT1D, and/or 5HT7 receptors interferes with their negative feedback actions, thus "cutting the brake cable" and thereby disinhibiting 5HT release (Figure 2C). ${ }^{13-23}$ Although the combination of SERT inhibition with 5HT1A agonism and simultaneous full or partial antagonism of 5HT1B, 5HT1D, and 5HT7 receptors causes more 5HT release than with SERT alone, the continuing presence of monoamine oxidase (MAO) activity ensures that 5HT levels do not rise to dangerous levels. Also, the same amount of 5HT release can be caused by lesser amounts of SERT inhibition when combined with these additional $5 \mathrm{HT}$ receptor actions. ${ }^{6,24}$ That is, most antidepressants acting predominantly by SERT inhibition have nearly complete blockade of the SERT at therapeutic doses, ${ }^{25-27}$ whereas SERT inhibition by vortioxetine at therapeutic doses can be as low as $50 \%{ }^{6,24}$ A plausible hypothesis to explain the apparently lesser amount of sexual dysfunction with vortioxetine compared to selective SERT inhibitors (SSRIs or selective serotonin reuptake inhibitors $)^{28}$ is this lower amount of SERT inhibition by vortioxetine ${ }^{6,24}$ combined with the other actions of vortioxetine at various $5 \mathrm{HT}$ receptors, causing enhanced release of $5 \mathrm{HT}$ in a manner that leads to antidepressant actions in some circuits but perhaps lack of sexual dysfunction in others. ${ }^{28}$

\section{Summary}

In summary, the multiple modes of action of vortioxetine, namely SERT inhibition with stimulation of 5HT1A presynaptic receptors plus blockade or partial blockade of presynaptic 5HT1B and 5HT1D receptors and postsynaptic 5HT7 receptors, combine to raise 5HT levels at multiple nodes within neural networks across the brain.

\section{References:}

1. Stahl SM. Classifying psychotropic drugs by mode of action and not by target disorder. CNS Spectr. 2013; 18(3): 113-117. 


\section{BRAINSTORMS - Clinical Neuroscience Update}

2. Stahl SM. Stahl's Essential Psychopharmacology, 4th ed. Cambridge, UK: Cambridge University Press; 2013.

3. Zohar J, Stahl S, Moller H-J, et al. Neuroscience Based Nomenclature. Cambridge, UK: Cambridge University Press; 2014.

4. Mork A, Pehrson A, Brennum LT, et al. Pharmacological effects of Lu AA21004: a novel multimodal compound for the treatment of major depressive disorder. J Pharmacol Exp Ther. 2012; 340(3): 666-675.

5. Bang-Andersen B, Ruhland T, Jorgensen M, et al. Discovery of 1-[2(2,4-dimethylphenylsulfanyl)phenyl]piperazine (Lu AA21004): a novel multimodal compound for the treatment of major depressive disorder. J Med Chem. 2011; 54(9): 3206-3221.

6. Areberg J, Luntang-Jensen M, Søgaard B, Nilausen DØ. Occupancy of the serotonin transporter after administration of Lu AA21004 and its relation to plasma concentration in healthy subjects. Basic Clin Pharmacol Toxicol. 2012; 110(4): 401-404.

7. Stahl SM. Modes and nodes explain the mechanism of action of vortioxetine, a multi-modal agent (MMA): modifying serotonin's downstream effects on glutamate and GABA (gamma-aminobutyric acid) release by blocking $5 \mathrm{HT} 3$ and $5 \mathrm{HT} 7$ receptors. CNS Spectr. In press.

8. Stahl SM. Modes and nodes explain the mechanism of action of vortioxetine, a multi-modal agent (MMA): enhancing downstream release of neurotransmitters by blocking $5 \mathrm{HT} 3$ receptors. CNS Spectr. In press.

9. Stahl SM. Modes and nodes explain the mechanism of action of vortioxetine, a multi-modal agent (MMA): enhancing downstream release of four pro-cognitive neurotransmitters by blocking 5HT1D receptors. CNS Spectr. In press.

10. Insel $\mathrm{T}$, Cuthbert $\mathrm{B}$, Garvey $\mathrm{M}$, et al. Research domain criteria (RDoC): toward a new classification framework for research on mental disorders. Am J Psychiatry. 2010; 167(7): 748-751.

11. Pehrson AL, Sanchez C. Serotonergic modulation of glutamate neurotransmission as a strategy for treating depression and cognitive dysfunction. CNS Spectr. 2014; 19(2): 121-133.

12. Pehrson AL, Cremers T, Betry C, et al. Lu AA21004, a novel multimodal antidepressant, produces regionally selective increases of multiple neurotransmitters-a rat microdialysis and electrophysiology study. Eur Neuropsychopharmacol. 2013; 23(2): 133-145.

13. Fink KB, Gothert M. $5 \mathrm{HT}$ receptor regulation of neurotransmitter release. Pharmacol Rev. 2007; 59(4): 360-417.

14. Assié MB, Lomentach $\mathrm{H}$, Ravailhe V, Faucillon V, NewmanTancredi A. Rapid desensitization of somatodendritic 5HT1A receptors by chronic administration of the high-efficacy 5HT1A agonist, F13714: a microdialysis study in the rat. Br J Pharmacol. 2006; 149(2): 170-178.

15. Gardier AM, Malgie I, Trillat AC, Jacquot C, Artigas F. Role of 5HT1A autoreceptors in the mechanism of action of serotoninergic antidepressant drugs: recent findings from in vivo microdialysis studies. Fundam Clin Pharmacol. 1996; 10(1): 16-27.

16. Romero L, Ben N, Artigas F, De Montigny C, Blier P. Effect of pindolol on the function of pre- and postsynaptic $5 \mathrm{HT} 1 \mathrm{~A}$ receptors: in vivo microdialysis and electrophysiological studies in the rat brain. Neuropsychopharmacology. 1996; 15(4): 349-360.

17. Sprouse JS, Aghajanian GK. Electrophysiological responses of serotoninergic dorsal raphe neurons to 5HT1A and 5HT1B agonists. Synapse. 1987; 1(1): 3-9.

18. Adell A, Celada P, Artigas F. The role of 5HT1B receptors in the regulation of serotonin cell firing and release in the rat brain. J Neurochem. 2001; 79(1): 172-182.

19. De Groote L, Klompmakers AA, Olivier B, Westenberg HG. Role of extracellular serotonin levels in the effect of 5HT1B receptor blockade. Psychopharmacology (Berl). 2003; 167(2): 153-158.

20. Ruf BM, Bhagwagar $Z$. The $5 \mathrm{HT} 1 \mathrm{~B}$ receptor: a novel target for the pathophysiology of depression. Curr Drug Targets. 2009; 10(11): 1118-1138.

21. Sari Y. Serotonin $1 B$ receptors: from protein to physiological function and behavior. Neurosci Biobehav Rev. 2004; 28(6): 565-582.

22. Sarkisyan G, Roberts AJ, Hedlund PB. The 5 HT7 receptor as a mediator and modulator of antidepressant-like behavior. Behav Brain Res. 2010; 209(1): 99-108.

23. Bonaventure P, Kelly L, Aluisio L, et al. Selective blockade of 5-hydroxytryptamine (5HT)7 receptors enhances 5HT neurotransmission, antidepressant-like behavior and rapid eye movement sleep suppression induced by citalopram in rodents. J Pharmacol Exp Ther. 2007; 321(2): 690-698.

24. Stenkrona P, Halldin C, Lundberg J. 5HTT and 5HT1A receptor occupancy of the novel substance vortioxetine (Lu AA21004). A PET study in control subjects. Eur Neuropsychopharmacol. 2013; 23(10): 1190-1198

25. Meyer JH. Imaging the serotonin transporter during major depressive disorder and antidepressant treatment. J Psychiatry Neuosci. 2007; 32(2): 86-102.

26. Lundberg J, Tiger M, Landén M, Halldin C, Farde L. Serotonin transporter occupancy with TCAs and SSRIs: a PET study in patients with major depressive disorder. Int $\mathrm{J}$ Neuropsychopharmacol. 2012; 15(8): 1167-1172.

27. Meyer JH, Wilson AA, Sagrati S, et al. Serotonin transporter occupancy of five selective serotonin reuptake inhibitors at different doses: an 11C-DASB positron emission tomography study. Am J Psychiatry. 2004; 161(5): 826-835.

28. Citrome L. Vortioxetine for major depressive disorder: a systematic review of the efficacy and safety profile for this newly approved antidepressant-what is the number needed to treat, number needed to harm and likelihood to be helped or harmed? Int J Clin Pract. 2014; 68(1): 60-82. 